\title{
Cultural heritage and awareness: differences between volunteered geographic information of OpenStreetMap and an official cartography. The case of Caserta in South-Italy
}

\author{
Giovanni Mauro $^{\mathrm{a}, *}$, Maria Ronza $^{\mathrm{b}}$ \\ a University of Campania “Luigi Vanvitelli”-giovanni.mauro@unicampania.it \\ ${ }^{b}$ University of Naples “Federico II"-mronza@unina.it \\ * Corresponding author
}

Keywords: Cultural Heritage, Participatory maps. OpenStreetMap, ICR Risk map, Caserta

\begin{abstract}
:
The representation of a place is becoming one of the most important research issues in the Geographic Information System (GIS) and in the participatory cartography (Hobel, Fogliaroni and Frank, 2016). As well known, in behavioural geography a mental map represents the personal perception of the surrounding geographical space (Lynch, 1960; Gould and White, 1974). Usually, these maps are very simple, so everyone can create them. The recent rise of internet and the advent of Web2.0 may mean, among others, the possibility for everyone to map. This is a sort of "revolution" in the cartography production, so now the geospatial data are often collected by people taking part in the generation of Volunteered Geographic Information, well known also as VGI (Goodchild, 2007). OpenStreetMap (OSM) is probably the most famous of these cartographic projects. Started in the UK in the early 2000s, now it provides free topographic maps at global scale (Ramm et al., 2011). Thus, in just a few decades, we have gone from mental maps to being able to collaborate on participative maps online (Panek, 2016). However, how much could knowledge of our living region affects the results of our online mapping activities? Namely, how much is important the personal perception of surrounding space in participatory cartography?
\end{abstract}

This research focuses on cultural heritage to recognize its important role in both local community identity (as "common identity heritage") and the tourism industry. In a region particularly rich in such unique features such as Campania Region (South Italy), awareness of its own identity heritage is sometimes lacking, leading to its degradation and neglect. The aim of this research is to investigate about the differences between the VGI of OSM and an official cartography of cultural heritage in the historical centre of Caserta, an important urban area of the Campania. This town is best known in Italy and abroad for its Royal Palace, a former royal residence built in the XVIII Century by the House of Bourbon-Two Sicilies and now one of the most visited monuments in Italy.Our sources of data are the cultural heritage risk map realized by the "Istituto Centrale per il Restauro del Ministero della cultura" (ICR) (High Institute for Conservation and Restoration, Ministry of Culture) and the PostgreSQL database of OSM. The first is a GIS project about Italian cultural heritage to assess the risk due to their state of preservation and the characteristics of the territory within they are (Bartolomucci, Cacace, 2008). Since the end of 2020, the General Directorate for Security of the Ministry of Culture manages the project. Currently the webGIS of the ICR map is freely available on http://www.cartadelrischio.beniculturali.it The OSM database, instead, is resulting from activities of VGI mapping geoinformation such as points or lines and polygons. The key component of OSM is its main database, which contains tables for each element type, where the attribute information of each object is referred to as a 'tag' (Ramm et al., 2011).Starting from the recent municipal urban plan of Caserta (Bottaro et al., 2017), we defined the border of the historical center of this town. We use this area to automatically extract the georeferenced data from ICR map and from the OMS database. In this last case, we use a QGIS plugin - QuickOSM plugin - to set up queries on the OSM database about buildings, areas, sites, monuments, churches and fountains. Then we compare our results to outline main differences between the official map (ICR map) and the crowdsourcing data of OSM. In detail, we examined the spatial distribution of cultural heritage in the study area and what is mapped by VGI.

The results put in evidence a great difference between the two selected sources of data: the ICR identifies 115 points, while OSM detects 22 points and 27 polygons. Considering the type of cultural good, the first map highlights a set very rich and heterogeneous: in the centre of Caserta there are 57 historical buildings, 13 churches, 8 chapels, 6 fountains, 3 theatres, 3 historical houses and other 21 elements. On the other side, the geographical information of OSM is a little more multi-faceted: it reports 7 historical palaces (all polygons), 16 ancient churches (15 polygons and 1 point, classified as chapel), 9 ancient and modern fountains (5 polygons and 4 points) and 17 "monuments" (all points, comprehensive of 
figures, commemorative plaques, artistic statues, etc.). Regarding their spatial distribution, the ICR map includes items within all the study area, even if their main concentration is close to the Royal Palace (fig. 1A). Concerning the OSM map, some areas of Caserta's historic centre are not even mapped in detail; they are mainly the historical neighbourhoods farthest from the Royal Palace, such as Ercole or Sala. Consequently, also the cultural heritage of OSM map is mostly located close to the Royal Palace or within its park (fig. 1B).
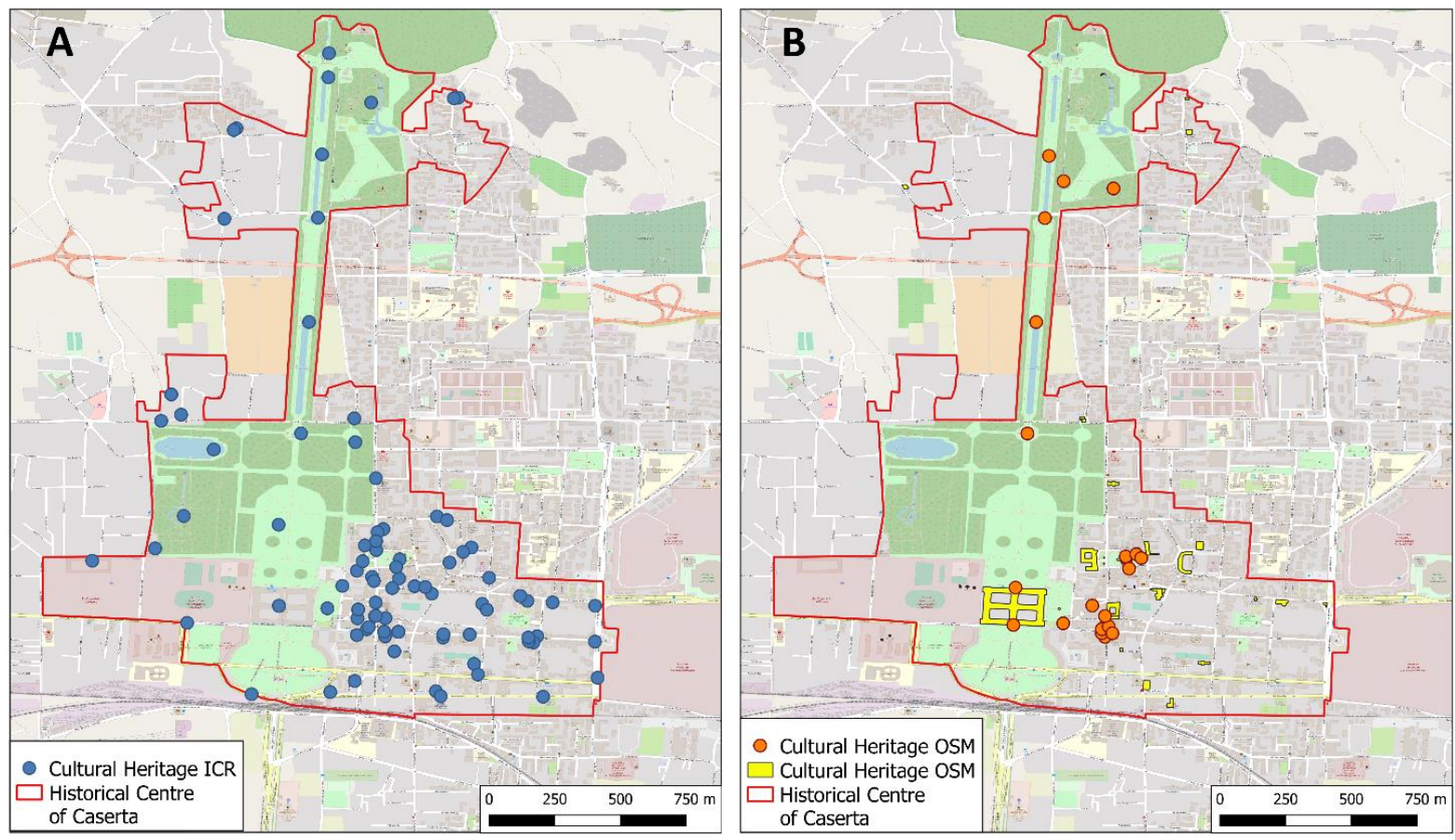

Figure 1. The spatial distribution of cultural heritage in the historical centre of Caserta: A) the ICR map; B) the OSM map (source: elaboration of Authors). Base map: OpenStreetMap.

As a conclusion, we can shortly report that the cultural heritage mapped by VGI of OSM is overall quite significant (about the $40 \%$ of total assets mapped by ICR). However, the results strictly depend on their typology: in our case, for instance, they are better for churches, but worse for historic palaces. Furthermore, the resulting OSM map is strongly influenced by the "bulky" presence in this city of such an important monument as the Royal Palace, as VGI mapped what is directly related or otherwise spatially close.

\section{Acknowledgements}

We acknowledge Dr Cacace of the "Istituto Centrale per il Restauro del Ministero della cultura" (ICR) for supporting this research by making the ICR map of risk available.

\section{References}

Bartolomucci, C., Cacace, C., 2008. La Carta del rischio del patrimonio culturale: normalizzazione delle tipologie degli edifici nella banca dati del Sistema informativo territoriale. Bollettino ICR, 16, pp. 69-77.

Bottaro, P., Pica Ciamarra, M., Realfonzo, A., Rocereto, A., De Lisa, S., Borrelli, E., Damiani, F., Albanese, G., 2017. Piano Urbanistico Comunale (PUC), Città di Caserta, Progetto Preliminare. Comune di Caserta.

Gould, P. and White, R., 1974. Mental maps. Harmondsworth: Pelican.

Goodchild, M.F., 2007. Citizen as sensors: the world of volunteered geography. GeoJournal, 69 (4), $211-221$.

Hobel, H., Fogliaroni, P., Frank, A.U., 2016. Deriving the geographic footprint of cognitive regions. Geospatial Data in a Changing World, Springer International Publishing, Cham, Switzerland, pp. 67-84.

Lynch, K., 1960. The image of the city. Technology Press, Cambridge, MA (USA).

Pánek, J., 2016. From Mental Maps to GeoParticipation. The Cartographic Journal 53 (4): 300-307.

Ramm, F., Topf, J., Chilton, S., 2011. OpenStreetMap. Using and enhancing the free map of the world. UIT, Cambridge (UK 